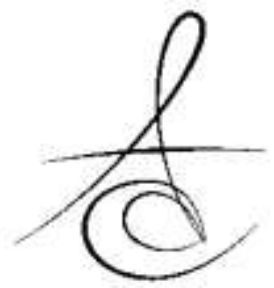

\title{
DETERMINATION OF THE KNOWLEDGE LEVEL OF ADULT PATIENTS ABOUT PERIODONTAL AND DENTAL HEALTH IN SOUTHEAST TURKEY: A CROSS SECTIONAL STUDY AND EPIDEMIOLOGICAL RESEARCH
}

\section{TÜRKİYE'NİN GÜNEYDOĞU BÖLGESİNDEKİ YETİŞKİN HASTALARIN PERİODONTAL VE DİŞ SAĞLIĞI HAKKINDAKİ BİLGİ DÜZEYİNİN BELİRLENMESİ}

\author{
Dr. Öğr. Üyesi Abdulsamet TANIK*
}

Makale Kodu/Article code: 4167

\section{ABSTRACT}

Aim: The study aims to determine the level of knowledge about periodontal and dental health and the prevalence of periodontal diseases of adult patients in the southeast region of Turkey.

Material and Method: The study included 10 thousand patients aged 18 to 77 years. To gather information about demographic characteristics, oral hygiene, oral care habits, and periodontal status, a questionnaire of 15 questions were applied to the participants. The probing pocket depth (PPD) of all patients participated in the research were measured from 4 surfaces of the teeth. The collected data was analyzed statistically.

Results: In this study, there was a statistically significant difference between gender and age and between those who went to dental doctors when had a toothache and those brushing teeth twice a day $(p<0.001)$. The proportion of the respondents to these two questions was the highest in women and 18-30 age groups. There was a significant difference between halitosis and gum bleeding and between periodontal condition and education of level $(p<0.001)$. The proportion of those who answered these two questions was found to be higher in those with periodontitis and high school-level education. In terms of periodontitis, men are at risk of 1.33 times more (95\% CI: $1.23-1.45$ ) compared to women, and age group of $44-56$ years is at risk of 1.73 times more ( $95 \% \mathrm{CI}: 1.41-2.53$ ) compared to the age group of $18-30$ years.

Conclusion: According to the survey results, the majority of participants have knowledge about periodontal and dental health, but we think that individuals do not perform oral care to maintain oral and dental health due to the high prevalence of periodontal disease. The validity of this questionnaire can be investigated in larger population field studies.

Keywords: Oral and Dental Health, Awareness, Adult, Periodontal Status

Öz

Amaç: Türkiye'nin Güneydoğu bölgesindeki yetişkin hastaların periodontal ve diş sağığı hakkındaki bilgi düzeyi ve periodontal hastalıkların prevalansı belirlenmesi amaçlanmıştır.

Materyal ve Metod: Çalışmaya 18-77 yaş arasındaki 10000 hasta dahil edilmiştir. Katılımcılara demografik özellikleri, oral hijyen ve ağız bakımı alışkanlıkları ve periodontal durum ile ilgili bilgi toplamak amacıyla, soru-cevap şeklinde yapılandırılmış 15 soruluk anket uygulanmıştır. Araştırmaya alınan bütün hastaların dişlerin 4 yüzeyinden sondalamada cep derinliği (SCD) ölçümü yapılmıştır. Toplanan veriler istatistiksel olarak analiz edilmiştir.

Bulgular: Bu çalışmada; cinsiyet ve yaş ile diş ağrısı olunca diş hekimine gidenler ve günde iki kez diş fırçalayanlar arasında istatistiksel olarak anlamlı bir fark olduğu $(p<0.001)$ ve en yüksek oranın kadınlar ve 18-30 yaş grubunda olduğu bulunmuştur. Ağız kokusu ve diş eti kanaması ile periodontal durum ve eğitim düzeyi arasında anlamlı fark olduğu( $p<0.001)$ ve bu iki soruyu cevaplayanların oranının periodontitis ve lise düzeyinde eğitim alanlarda yüksek olduğu bulunmuştur. Periodontitis açısından, erkekler kadınlara göre 1.33 kat daha fazla (\% 95 CI: 1.23-1.45), 44-56 yaş grubu ise 18-30 yaş grubuna kıyasla 1.73 kat daha fazla (\% 95 CI: 1.41-2.53) risk altında bulunmuştur.

Sonuç: Anket sonuçlarına göre, ankete katılanların büyük çoğunluğu periodontal ve diş sağlığının hakkında bilgili olduğunun farkındadır, fakat periodontal hastalık prevalansının yüksek çıkmasından dolayı bireylerin ağız ve diş sağı̆̆ını korumak için yapmaları gereken ağız bakımını yapmadıkları düşünmekteyiz. Daha büyük popülasyonlu saha çalışmalarında bu anketin geçerliliği araştırılabilir.

Anahtar kelimeler: Ağız ve diş sağlığı, Farkındalık, Yetişkin, Periodontal durum

\footnotetext{
${ }^{*}$ Department of Periodontology, Faculty of Dentistry, Adıyaman University, Adıyaman.
} 


\section{INTRODUCTION}

Periodontal diseases, tooth decay, tooth loss, and oral diseases, are a serious public health problem that is still widely seen in the world. Oral and dental health $(\mathrm{ODH})$ is an important part of general health. Systemic diseases such as diabetes, cardiovascular diseases, respiratory system diseases, rheumatoid arthritis are known to affect $\mathrm{ODH}^{1}$.

$\mathrm{ODH}$ are among the almost entirely preventable and treated diseases, but it has some adverse effects on human health. Moreover, the treatment of these diseases is expensive and leads to loss of labor. There has been a positive improvement in the development of health programmes and policies to improve $\mathrm{ODH}$ across countries recently ${ }^{2}$.

The emphasis on preventive measures and the importance of dental health in ODH play an important role in raising awareness of the community 3 . Childhood education is gaining importance in raising awareness of $\mathrm{ODH}$ in societies. Dentists have an important role in this regard. Health workers other than dentists also have information about $\mathrm{ODH}$ and they do present accurate information on the community.

Oral hygiene education and preventive programs are known to be of critical importance in reducing the prevalence of caries and periodontal disease in adults ${ }^{4}$. It has been reported that the role of parents, teachers, and friends is more important than dentists in the promotion of oral hygiene in adults ${ }^{5}$. The source of information related to $\mathrm{ODH}$ in Turkey was determined as parents with $35 \%$, individuals with $19 \%$, dentists with $18 \%$, TV with $15 \%$ and school with $13 \%$. The level of knowledge on the $\mathrm{ODH}$ in society is very limited. The task of tackling $\mathrm{ODH}$ problems, which is a preventable disease, should not be left only to dentists, whose numbers are 22,000 in Turkey, $71 \%$ of whom are known to work in the private sector ${ }^{6}$.

Although microbial dental plaque is considered to be the primary factor in the etiology of periodontal disease and tooth decay, it was also reported that $\mathrm{ODH}$ levels may vary according to the oral hygiene habits, systemic diseases, education level and demographic status of individuals ${ }^{7,8}$.

There is a decrease in the prevalence of tooth decay in developed countries, although an increase is seen in developing countries. When the average of all age groups is taken in our country, $92 \%$ of people need treatment due to tooth decay. In the 20-24 age group, 5.48 teeth on average should be treated. In the 25-29 age group, this number is 7 . The prevalence of caries in the $30-35$ age group is $97 \%$. The incidence of periodontal diseases is $50 \%$ at the age of 10 , while $96 \%$ at advanced ages. ${ }^{6}$.

The aim of this study is to determine some demographic characteristics that may be associated with oral and dental health in adults in the southeastern region of Turkey, also, to identify the prevalence of periodontal disease and to determine the level of knowledge about systemic conditions by the survey on $\mathrm{ODH}$.

\section{MATERIALS AND METHODS}

\section{Individuals}

This study is cross-sectional and epidemiological research, which is based on the permission of the Ethics Committee of the Faculty of Medicine, Adiyaman University (Protocol no:2018/8-2). The study group included a total of 10 thousand patients (5212 women and 4788 men) who are in the ages of 18-77 and randomly selected between 2018 2019 years. Prior to the application, the objectives of the research were clarified to the participants, and the participation in the research was explained to be volunteer-based, and informed consent forms were taken from all the participants. This study was conducted in the Faculty of Dentistry of Diyarbakır, Şanlıurfa and Gaziantep provinces, especially in the Periodontology Clinic of Adıyaman University in the southeast region of Turkey. Clinical measurements were performed to determine the periodontal status of individuals. To obtain knowledge levels about oral care habits and systemic status, and to determine their demographic data, a questionnaire was filled by individuals with the help of researchers. Statistical analyses of the individuals participating in the study were made according to the age groups of $18-30,31-$ 43, 44-56, 57-69, and 70-82.

\section{Clinical intraoral examination and questionnaire filling}

In the study, clinical examinations of patients were performed by 3 dentists. The questions in the questionnaire were filled by the dentist by asking and explaining the statements to the patients face to face. To standardize the examinations and questionnaire filling, a directive was set up by the dentists themselves. After that, each dentist made 
examinations of at least 10 people of each age group within a week and completed the questionnaire. Then, the examination results of the dentists were compared and calibrated at least at the level of $85 \%$.

\section{Survey Investigation}

This questionnaire was conceptually designed by a literature review. Information about $\mathrm{ODH}$ was determined through literature search ${ }^{9-11}$. To measure the knowledge of the individuals involved in the study about oral and dental health, they were asked to answer a survey of 15 questions. In the first step of the survey, demographic data (age, gender, and education status) was questioned, and in the second step, the information on oral care habits (tooth brushing method, brushing duration, auxiliary toothcleaning tools, awareness of gingiva disease, dentist visits, nutrition, tooth decay, antibiotic use, and halitosis), information on systemic status (smoking and systemic disease) were included in the survey.

\section{Periodontal Examination}

In our research, we measured PPD from 4 surfaces of each tooth with a complete oral examination, and the regions with PPD $\geq 4 \mathrm{~mm}$ were recorded. Patients with PPD $\geq 4 \mathrm{~mm}$ at least one tooth and with the loss of alveolar bone and attachment were evaluated as periodontitis; Patients with PPD $\leq 4 \mathrm{~mm}$ and without loss of alveolar bone and attachment were evaluated as gingivitis ${ }^{11}$. It was performed by 3 dentists (x. x. x) calibrated for PPD measurement by using Williams probes (Hu-friedy, Chicago, IL).

\section{Statistical analysis}

The data were evaluated with SPSS 15.0 package software (SPSS, Chicago, IL, USA) in the computer environment. The descriptive statistics were presented as averages, standard deviations (SD) and percentage distributions. In the parametric analyses, the chi-square test was used to investigate the relationship between categorical variables that are normally distributed. Logistic regression analysis was used in the multivariate evaluation of risk factors affecting periodontitis diagnosis. Statistical significance level was accepted as $\mathrm{p}<0.05$.

\section{RESULTS}

\section{Demographic data}

The distribution of gender, age, education and presence of periodontal disease of the patients involved in the study groups is shown in Table 1 . The study group included a total of 10 thousand patients (5212 women and 4788 men) between the ages of 18-77 (33.84 11.70$)$. The majority of the participants are graduated in high school and university $(31.0 \%$ and $27.9 \%$, respectively). The clinical measurements revealed that $3572(35.7 \%)$ people had gingivitis, and $6428(64.3 \%)$ people had periodontitis (Table 1 ).

Tablo 1. Demographic characteristics of subjects

\begin{tabular}{|c|c|c|c|c|}
\hline Variable & $\begin{array}{l}\text { Categories( } \\
\text { n) }\end{array}$ & $\begin{array}{l}\text { Percent( } \\
\%)\end{array}$ & $\underset{*}{\operatorname{Mean} \pm S D}$ & $\begin{array}{l}\text { P- } \\
\text { Value }\end{array}$ \\
\hline $\begin{array}{l}\text { Total } \\
\text { Sample }\end{array}$ & 10000 & 100.0 & & \\
\hline \multirow{3}{*}{$\begin{array}{l}\text { Gender } \\
\text { Male } \\
\text { Female }\end{array}$} & & & & $\begin{array}{l}<0.00 \\
1\end{array}$ \\
\hline & 4788 & 47.9 & & \\
\hline & 5212 & 52.1 & & \\
\hline $\begin{array}{l}\begin{array}{l}\text { Age } \\
\text { (years) }\end{array} \\
\end{array}$ & & & $\begin{array}{l}33.84 \pm 11 . \\
70\end{array}$ & $\begin{array}{l}<0.00 \\
1\end{array}$ \\
\hline $18-30$ & 4225 & 42.3 & & \\
\hline $31-43$ & 3524 & 35.2 & & \\
\hline 44-56 & 1832 & 18.3 & & \\
\hline $57-69$ & 398 & 4.0 & & \\
\hline $70-82$ & 21 & 0.2 & & \\
\hline $\begin{array}{l}\text { Level of } \\
\text { Education }\end{array}$ & & & & $\begin{array}{l}<0.00 \\
1\end{array}$ \\
\hline $\begin{array}{l}\text { None } \\
\text { (pre- } \\
\text { school) }\end{array}$ & 419 & 4.2 & & \\
\hline $\begin{array}{l}\text { Elementar } \\
\text { y school }\end{array}$ & 2057 & 20.6 & & \\
\hline $\begin{array}{l}\text { Middle } \\
\text { school }\end{array}$ & 1376 & 13.8 & & \\
\hline $\begin{array}{c}\text { High } \\
\text { school }\end{array}$ & 3097 & 31.0 & & \\
\hline University & 2786 & 27.9 & & \\
\hline $\begin{array}{l}\text { Phd or } \\
\text { Above }\end{array}$ & 265 & 2.7 & & \\
\hline $\begin{array}{l}\text { Periodonta } \\
\text { I Status } \\
\end{array}$ & & & & $\begin{array}{l}<0.00 \\
1\end{array}$ \\
\hline Gingivitis & 3572 & 35.7 & & \\
\hline $\begin{array}{l}\text { Periodontit } \\
\text { is }\end{array}$ & 6428 & 64.3 & & \\
\hline
\end{tabular}

The data were analysed using $X^{2}$ tests, $\mathrm{P}<0.05$.

*Standard Deviation.

\section{Answers to survey questions about ODH}

The answers to the questionnaire that measure information levels of patients about ODH are shown in Table 2. Patients included in the study were asked whether they agreed on the statement "If the brushing method of teeth is not done correctly, oral and dental health is affected negatively?", and $88.2 \%$ of whom stated to agree on it, $6.5 \%$ didn't agree and $3.3 \%$ stated not to have any idea. Patients were asked whether they agreed on the statement "Teeth should be brushed for 2 minutes at least 2 times a day?", and $80.4 \%$ of whom stated to agree on it, $10.3 \%$ didn't agree. When asked whether they agreed on the

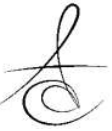


statement "Using auxiliary tooth-cleaning tools other than toothbrushes has the benefits of reducing tooth decay and gingival disease?", $37.4 \%$ of whom stated to agree on it, $24.7 \%$ didn't agree. When asked whether they agreed on the statement "It is enough to use only antibiotics when toothache or gingivitis occurs?", $25.3 \%$ of whom stated to agree on it, $33.8 \%$ didn't agree. When asked whether they agreed on the statement "The cause of dental caries and gingival diseases is microorganisms?", $48.9 \%$ of whom stated to agree on it, $11.2 \%$ didn't agree and $14.8 \%$ stated not to have any idea. It was found that $89 \%$ of the patients agreed on the statement "Smoking has negative effects on oral and teeth health". When asked whether they agreed on the statement "Gingival bleeding is the most important symptom of gingival disease?", and "The most important way to prevent dental caries and gingival disease is to brush teeth?", the rates of those stated to agree were $74.8 \%$ and $84 \%$, respectively. When asked whether they agreed on the statement "Diseases such as hepatitis B and
AIDS may give symptoms in the mouth?", $23 \%$ of whom stated to agree on it, and $38.5 \%$ stated not to have any idea. When asked whether they agreed on the statement "Halitosis may be a symptom of a problem in the mouth?", $73.6 \%$ of whom stated to agree on it. Also, $69.9 \%$ of the patients stated to agree on the statement "Nutrition has an effect on oral and dental health?", and $73.9 \%$ of whom stated to agree on the statement "Regular oral and dental examinations should be done at least six-monthly intervals?" (Table 2).

The distribution of the answers to questions about the ODH was given in Table 3 and a statistically significant difference was found between the gender and age groups $(P<0.05)$. The women and individuals in the 18-30 age group agreed mostly on the following statements; "if the brushing method of teeth is not done correctly, oral and dental health is affected negatively?", "Using auxiliary cleaning tools other than toothbrushes has the benefits of reducing

Table 2. Distribution of patients' answers to survey problems

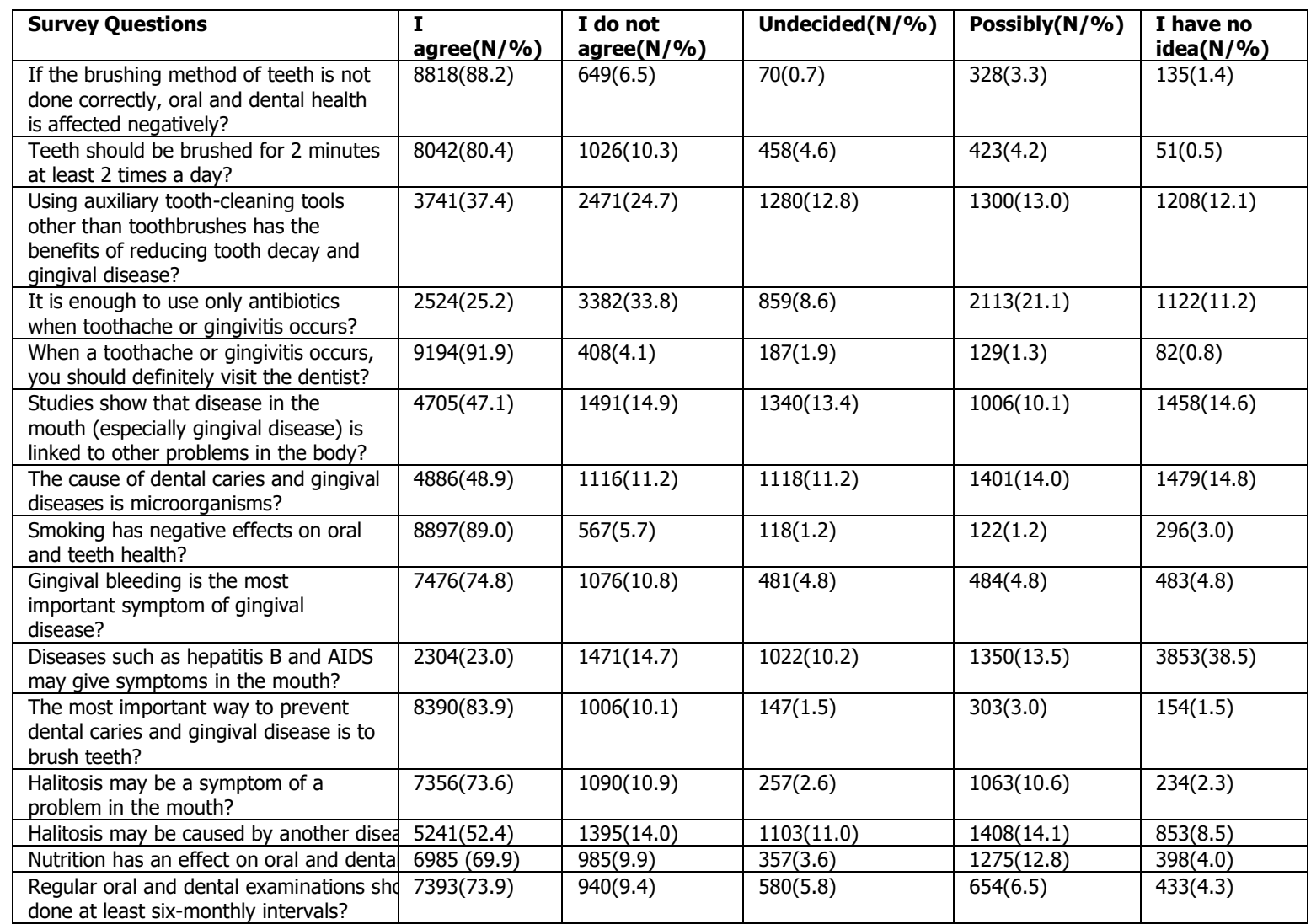


tooth decay and gingival disease?", "When a toothache or gingivitis occurs, you should definitely visit the dentist?", "smoking has negative effects on oral and teeth health?", "Gingival bleeding is the most important symptom of gingival disease?", "The most important way to prevent dental caries and gingival disease is to brush teeth?", "Halitosis may be a symptom of a problem in the mouth?", "Halitosis may be caused by another disease?", "Nutrition has an effect on oral and dental health?", "Regular oral and dental examinations should be done at least 6 months intervals?" The women and individuals in the 31-43 age group agreed frequently on the statement "It is enough to use only antibiotics when toothache or gingivitis occurs?". Also, the men and individuals in the 31-43 age group agreed generally on the statement "Studies show that disease in the mouth (especially gingival disease) is linked to other problems in the body?". The men and individuals in the 31-43 age group agreed generally on the statement "The cause of dental caries and gingival diseases is microorganisms?", also, the women and individuals in the 18-30 age group stated generally not to have any idea about the statement "Diseases such as hepatitis B and AIDS may give symptoms in the mouth?" (Table 3).

Table 3. Comparison of gender and age groups along with the answers of patients to questions

\begin{tabular}{|c|c|c|c|c|c|c|c|c|c|}
\hline \multirow{2}{*}{ Survey Questions } & \multicolumn{2}{|c|}{ Gender N(\%) } & \multirow{2}{*}{$P$ Value } & \multicolumn{5}{|c|}{ Age Groups N(\%) } & \multirow{2}{*}{ P Value } \\
\hline & Male & Female & & $18-30$ & $31-43$ & $44-56$ & $57-69$ & $70-82$ & \\
\hline I agree & $4245(42.5)$ & $4577(45.8)$ & \multirow[t]{3}{*}{$<0.001$} & $3688(36.9)$ & $3172(31.7)$ & $1596(16)$ & $348(3.5)$ & $19(0.2)$ & \multirow[t]{3}{*}{$<0.001$} \\
\hline Possibly & $71(0.7)$ & $254(2.5)$ & & $262(2.6)$ & $27(0.3)$ & $25(0.3)$ & $10(0.1)$ & $1(0)$ & \\
\hline I have no idea & $20(0.2)$ & $115(1.2)$ & & $30(0.3)$ & $100(1)$ & $5(0.1)$ & 0 & 0 & \\
\hline \multicolumn{10}{|c|}{ Teeth should be brushed for 2 minutes at least 2 times a day? } \\
\hline Undecided & $382(3.8)$ & $76(0.8)$ & \multirow{3}{*}{$<0.001$} & $162(1.6)$ & $250(2.5)$ & $30(0.3)$ & $15(0.2)$ & $1(0)$ & \multirow{3}{*}{$<0.001$} \\
\hline Possibly & $311(3.1)$ & $110(1.1)$ & & $205(2.1)$ & $61(0.6)$ & $125(1.3)$ & $29(0.3)$ & $1(0)$ & \\
\hline I have no idea & $20(0.2)$ & $30(0.3)$ & & $10(0.1)$ & $30(0.3)$ & $10(0.1)$ & 0 & 0 & \\
\hline \multicolumn{10}{|c|}{ Using auxiliary tooth-cleaning tools other than toothbrushes has the benefits of reducing tooth decay and gingival disease? } \\
\hline I agree & $1625(16.3)$ & $2119(21.2)$ & \multirow[t]{2}{*}{$<0.001$} & $1615(16.2)$ & $1224(12.2)$ & $822(8.2)$ & $83(0.8)$ & 0 & \multirow[t]{2}{*}{$<0.001$} \\
\hline I do not agree & $1310(13.1)$ & $1159(11.6)$ & & $1005(10.1)$ & $719(7.2)$ & $475(4.8)$ & $270(2.7)$ & 0 & \\
\hline I agree & $1265(12.7)$ & $1260(12.6)$ & \multirow[t]{5}{*}{$<0.001$} & $910(9.1)$ & $1045(10.5)$ & $521(5.2)$ & $49(0.5)$ & 0 & \multirow[t]{5}{*}{$<0.001$} \\
\hline I do not agree & $1389(13.9)$ & 1994(19.9) & & $1170(11.7)$ & $1288(12.9)$ & $695(7)$ & $230(2.3)$ & 0 & \\
\hline Undecided & $518(5.2)$ & $340(3.4)$ & & $380(3.8)$ & $326(3.3)$ & $116(1.2)$ & $35(0.4)$ & $1(0)$ & \\
\hline Possibly & $1189(11.9)$ & $925(9.3)$ & & $1125(11.3)$ & $616(6.2)$ & $309(3.1)$ & $64(0.6)$ & 0 & \\
\hline I have no idea & $427(4.3)$ & $693(6.9)$ & & $640(6.4)$ & $249(2.5)$ & 191(1.9) & $20(0.2)$ & $20(0.2)$ & \\
\hline \multicolumn{10}{|c|}{ When a toothache or gingivitis occurs, you should definitely visit the dentist? } \\
\hline I agree & $4222(42.2)$ & $4976(49.8)$ & \multirow[t]{5}{*}{$<0.001$} & $3843(38.4)$ & $3212(32.1)$ & $1760(17.6)$ & $361(3.6)$ & $20(0.2)$ & \multirow[t]{5}{*}{$<0.001$} \\
\hline I do not agree & $276(2.8)$ & $133(1.3)$ & & $91(0.9)$ & $235(2.4)$ & $56(0.6)$ & $26(0.3)$ & $1(0)$ & \\
\hline Undecided & $155(16)$ & $31(0.3)$ & & $146(1.59$ & $30(0.3)$ & $5(0.2)$ & $5(0.2)$ & 0 & \\
\hline Possibly & $112(1.1)$ & $17(0.2)$ & & $105(1.1)$ & $17(0.2)$ & $6(0.1)$ & $1(0)$ & 0 & \\
\hline I have no idea & $25(0.3)$ & $55(0.6)$ & & $40(0.4)$ & $30(0.3)$ & $5(0.2)$ & $5(0.2)$ & 0 & \\
\hline \multicolumn{10}{|c|}{ Studies show that disease in the mouth (especially gingival disease) is linked to other problems in the body? } \\
\hline I agree & $2661(26.6)$ & $2046(20.5)$ & $<0.001$ & $1679(16.8)$ & $1799(18)$ & $906(9.1)$ & $313(3.2)$ & $10(0.1)$ & $<0.001$ \\
\hline Possibly & $617(6.2)$ & $783(7.8)$ & & $696(7)$ & $476(4.8)$ & $116(1.2)$ & 111(1.1) & $1(0)$ & \\
\hline I have no idea & $500(5)$ & $978(9.8)$ & & $505(5.1)$ & $624(6.2)$ & 291(2.9) & $49(0.5)$ & $9(0.1)$ & \\
\hline
\end{tabular}


Tablo 3'ün devamı

\begin{tabular}{|c|c|c|c|c|c|c|c|c|c|}
\hline \multicolumn{10}{|c|}{ Smoking has negative effects on oral and teeth health? } \\
\hline I agree & $4209(42.1)$ & $4692(46.9)$ & \multirow[t]{5}{*}{0.008} & $3864(38.6)$ & $3041(30.4)$ & $1711(17.1)$ & $267(2.7)$ & $18(0.2)$ & \multirow[t]{5}{*}{$<0.001$} \\
\hline I do not agree & $310(3.1)$ & $257(2.6)$ & & $201(2)$ & $210(2.1)$ & $45(0.5)$ & $111(1.1)$ & 0 & \\
\hline Undecided & $61(0.6)$ & $56(0.6)$ & & $60(0.6)$ & $10(0.1)$ & $35(0.4)$ & $11(0.1)$ & $1(0)$ & \\
\hline Possibly & $63(0.6)$ & $57(0.6)$ & & $70(0.7)$ & $33(0.3)$ & $15(0.2)$ & 0 & $2(0)$ & \\
\hline I have no idea & $145(1.5)$ & $150(1.5)$ & & $30(0.3)$ & $230(2.3)$ & $26(0.3)$ & $9(0.1)$ & 0 & \\
\hline \multicolumn{10}{|c|}{ Gingival bleeding is the most important symptom of gingival disease? } \\
\hline I agree & $3385(33.9)$ & $4094(40.9)$ & \multirow[t]{5}{*}{$<0.001$} & $2918(29.1)$ & $2817(28.1)$ & $1501(15)$ & $233(2.3)$ & $10(0.1)$ & \multirow[t]{5}{*}{$<0.001$} \\
\hline I do not agree & $750(7.5)$ & $326(3.3)$ & & $460(4.6)$ & $280(2.8)$ & $200(2)$ & $135(1.4)$ & $1(0)$ & \\
\hline Undecided & $236(2.4)$ & $244(2.4)$ & & $380(3.8)$ & $30(0.3)$ & $45(0.5)$ & $15(0.2)$ & $10(0.1)$ & \\
\hline Possibly & $185(1.9)$ & $298(3)$ & & $261(2.6)$ & $201(2)$ & $15(0.2)$ & $6(0.1)$ & 0 & \\
\hline I have no idea & $232(2.3)$ & $250(2.5)$ & & $206(2.1)$ & $196(2)$ & $71(0.7)$ & $9(0.1)$ & 0 & \\
\hline \multicolumn{10}{|c|}{ Diseases such as hepatitis B and AIDS may give symptoms in the mouth? } \\
\hline I agree & $1101(11)$ & $1204(12)$ & \multirow[t]{5}{*}{$<0.001$} & $959(9.6)$ & $869(8.7)$ & $391(3.9)$ & $85(0.9)$ & $1(0)$ & \multirow[t]{5}{*}{0.01} \\
\hline I do not agree & $879(8.8)$ & 592(5.9) & & $455(4.6)$ & $564(5.6)$ & $340(3.4)$ & 104(1) & $8(0.1)$ & \\
\hline Undecided & $497(5)$ & $526(5.3)$ & & $619(6.2)$ & $238(2.4)$ & $110(1.1)$ & $45(0.5)$ & $11(0.1)$ & \\
\hline Possibly & $890(8.9)$ & $459(4.6)$ & & $510(5.1)$ & $709(7.1)$ & $25(0.3)$ & $105(1.1)$ & 0 & \\
\hline I have no idea & $1421(14.2)$ & $2431(24.3)$ & & $1682(16.8)$ & $1144(11.4)$ & $966(9.7)$ & $59(0.6)$ & $1(0)$ & \\
\hline \multicolumn{10}{|c|}{ The most important way to prevent dental caries and gingival disease is to brush teeth? } \\
\hline I agree & $3729(37.3)$ & $4662(46.6)$ & \multirow[t]{5}{*}{$<0.001$} & $3580(35.8)$ & $3006(30.1)$ & $1435(14.4)$ & $351(3.5)$ & $19(0.2)$ & \multirow[t]{5}{*}{0.002} \\
\hline I do not agree & $711(7.1)$ & $296(3)$ & & $395(4)$ & $335(3.4)$ & $250(2.5)$ & $26(0.3)$ & $1(0)$ & \\
\hline Undecided & $97(1)$ & $50(0.5)$ & & $65(0.7)$ & $41(0.4)$ & $26(0.3)$ & $15(0.2)$ & 0 & \\
\hline Possibly & $236(2.4)$ & $66(0.7)$ & & $170(1.7)$ & $31(0.3)$ & $100(1)$ & 0 & $1(0)$ & \\
\hline I have no idea & $15(0.2)$ & $138(1.4)$ & & $15(0.2)$ & 111(1.1) & $21(0.2)$ & $6(0.1)$ & 0 & \\
\hline \multicolumn{10}{|c|}{ Halitosis may be a symptom of a problem in the mouth? } \\
\hline I agree & $3220(32.2)$ & $4140(41.4)$ & \multirow[t]{5}{*}{$<0.001$} & $3209(32.1)$ & $2523(25.2)$ & $1466(14.7)$ & $142(1.5)$ & $20(0.2)$ & \multirow[t]{5}{*}{$<0.001$} \\
\hline I do not agree & $635(6.4)$ & $455(4.6)$ & & $500(5)$ & $309(3.1)$ & $150(1.5)$ & $131(1.3)$ & 0 & \\
\hline Undecided & $107(1.1)$ & $150(1.5)$ & & $105(1.1)$ & 101(1) & $45(0.5)$ & $5(0.1)$ & $1(0)$ & \\
\hline Possibly & $730(7.3)$ & $331(3.3)$ & & $306(3.1)$ & $505(5.1)$ & 135(1.4) & $115(1.2)$ & 0 & \\
\hline I have no idea & $96(1)$ & $136(1.4)$ & & $105(1.1)$ & $86(0.9)$ & $36(0.4)$ & $5(0.1)$ & 0 & \\
\hline \multicolumn{10}{|c|}{ Halitosis may be caused by another disease? } \\
\hline I agree & $2317(23.2)$ & $2927(29.3)$ & \multirow[t]{5}{*}{$<0.001$} & $2084(20.8)$ & $1868(18.7)$ & $1075(10.8)$ & $199(2)$ & $18(0.2)$ & \multirow[t]{5}{*}{$<0.001$} \\
\hline I do not agree & $801(8)$ & $594(5.9)$ & & $750(7.5)$ & $390(3.9)$ & $225(2.3)$ & $30(0.3)$ & 0 & \\
\hline Undecided & $492(4.9)$ & $611(6.1)$ & & $391(3.9)$ & $436(4.4)$ & $255(2.6)$ & $20(0.2)$ & $1(0)$ & \\
\hline Possibly & 902(9) & $505(5.1)$ & & $520(5.2)$ & $560(5.6)$ & $211(2.1)$ & $115(1.2)$ & $1(0)$ & \\
\hline I have no idea & $276(2.8)$ & $575(5.8)$ & & $480(4.8)$ & $270(2.7)$ & $66(0.7)$ & $34(0.3)$ & $1(0)$ & \\
\hline \multicolumn{10}{|c|}{ Nutrition has an effect on oral and dental health? } \\
\hline I agree & $3321(33.2)$ & $3668(36.7)$ & \multirow[t]{5}{*}{$<0.001$} & $3099(31)$ & $2537(25.4)$ & $1241(12.4)$ & $103(1)$ & $9(0.1)$ & $<0.001$ \\
\hline I do not agree & $663(6.6)$ & $322(3.2)$ & & $331(3.3)$ & $334(3.3)$ & $160(1.6)$ & $150(1.5)$ & $10(0.1)$ & \\
\hline Undecided & $163(1.6)$ & $193(1.9)$ & & $175(1.8)$ & $105(1.1)$ & $60(0.6)$ & $14(0.1)$ & $2(0)$ & \\
\hline Possibly & $501(5)$ & $772(7.7)$ & & $360(3.6)$ & $442(4.4)$ & $365(3.7)$ & $106(1.1)$ & 0 & \\
\hline I have no idea & $140(1.4)$ & $257(2.6)$ & & $260(2.6)$ & $106(1.1)$ & $6(0.1)$ & $25(0.2)$ & 0 & \\
\hline Regular oral an & tal examina & done at leas & k-montr & ntervals? & & & & & \\
\hline I agree & $3335(33.4)$ & $4060(40.6)$ & $<0.001$ & $3184(31.8)$ & $2512(25.1)$ & $1491(14.9)$ & $189(1.9)$ & $19(0.2)$ & $<0.001$ \\
\hline I do not agree & $579(5.8)$ & $361(3.6)$ & & $446(4.6)$ & $244(2.4)$ & $219(2.2)$ & $30(0.3)$ & $1(0)$ & \\
\hline Undecided & $431(4.3)$ & $149(1.5)$ & & $265(2.7)$ & $251(2.5)$ & $40(0.4)$ & $24(0.2)$ & 0 & \\
\hline Possibly & $301(3)$ & $352(3.5)$ & & $60(0.6)$ & $420(4.2)$ & $37(0.4)$ & $136(1.4)$ & 0 & \\
\hline I have no idea & $142(1.4)$ & $290(2.9)$ & & $270(2.7)$ & $97(1)$ & $45(0.5)$ & $19(0.2)$ & $1(0)$ & \\
\hline
\end{tabular}

As shown in table 4, when the answers to survey questions related to $\mathrm{ODH}$ were evaluated, a statistically significant difference was found between the answers and the periodontal status, as well as, education levels $(P<0.05)$. Patients with periodontitis who participated in the survey stated that they agreed on all questions in a higher rate except the statement "Diseases such as hepatitis B and AIDS may give symptoms in the mouth?". Those who graduated from high school and university agreed on most of the survey questions (especially those graduated from high school) (Table 4).

The independent variables considered to affect the periodontal condition were included in multiple regression analysis. The risk factors affecting the periodontal condition were examined. The results of the logistic model were created with the statistical significance of these variables. According to this model, the independent risk factors affecting the periodontal condition are shown in Table 5 .

Male patients were found to be at 1.33 times more risk (OR: $1.33,95 \% \mathrm{CI}: 1.23-1.45$ ) in terms of periodontitis compared to female patients. Those between the ages of $31-43$ were found to be at 1.64 times more risk (OR: 1.64, 95\% CI: 1.13-2.01) in terms of periodontitis compared to the 18-30 years of age, and those between the ages of 44-56 and 57-69 were found to be at more risk (OR:1.73, \%95 CI:1.412.53, OR:1.71, \%95 CI:1.33-2.35, respectively) compared to the $18-30$ years of age. Those between the ages of $70-83$ were found to be at 2.1 times more risk (OR:2.1, \%95 CI:0.78-5.20) in terms of periodontitis compared to the $18-30$ years of age. 
Table 4. Comparison of education level and periodontal status along with the answers of the patients to the questions

\begin{tabular}{|c|c|c|c|c|c|c|c|c|c|c|}
\hline \multirow{2}{*}{$\begin{array}{l}\text { Survey } \\
\text { Questions }\end{array}$} & \multicolumn{2}{|c|}{ Periodontal Status } & \multirow[t]{2}{*}{ P Value } & \multicolumn{6}{|c|}{ Level of Education } & \multirow[t]{2}{*}{ P Value } \\
\hline & Gingivitis & Periodontitis & & $\begin{array}{l}\text { None (pre- } \\
\text { school) }\end{array}$ & $\begin{array}{c}\text { Elementary } \\
\text { school }\end{array}$ & $\begin{array}{l}\text { Middle } \\
\text { school }\end{array}$ & $\begin{array}{c}\text { High } \\
\text { school }\end{array}$ & University & $\begin{array}{l}\text { Phd or } \\
\text { Above }\end{array}$ & \\
\hline \multicolumn{11}{|c|}{ If the brushing method of teeth is not done correctly, oral and dental health is affected negatively? } \\
\hline I agree & $3183(31.8)$ & $5639(56.4)$ & \multirow[t]{5}{*}{$P<0.001$} & $267(2.7)$ & 1839(18.4) & $1185(11.9)$ & $2720(27.2)$ & $2564(25.6)$ & $243(2.4)$ & $P<0.001$ \\
\hline I do not agree & $312(3.1)$ & $336(3.4)$ & & $96(1)$ & $151(1.5)$ & $160(1.6)$ & $121(1.2)$ & $106(1.1)$ & $15(0.2)$ & \\
\hline Undecided & $25(0.3)$ & $45(0.5)$ & & $35(0.4)$ & $10(0.1)$ & $10(0.1)$ & $5(0.1)$ & $10(0.1)$ & 0 & \\
\hline Possibly & $42(0.4)$ & $283(2.8)$ & & $16(0.2)$ & $47(0.5)$ & $11(0.1)$ & $141(1.4)$ & $106(1.1)$ & $7(0.1)$ & \\
\hline I have no idea & $10(0.1)$ & $125(1.6)$ & & $5(0.1)$ & $10(0.1)$ & $10(0.1)$ & $110(1.1)$ & 0 & 0 & \\
\hline & & & should be & brushed for $2 \mathrm{n}$ & inutes at least & 2 times a day? & & & & \\
\hline I agree & $2880(28.8)$ & $5165(51.7)$ & 0.001 & $213(2.1)$ & $1264(12.6)$ & 1181(11.8) & 2671(26.7) & $2460(24.6)$ & $253(2.5)$ & $P<0.001$ \\
\hline I do not agree & $355(3.6)$ & $671(6.7)$ & & $111(1.1)$ & $435(4.4)$ & $90(0.9)$ & $225(2.3)$ & $160(1.6)$ & $5(0.1)$ & \\
\hline Undecided & $97(1)$ & $361(3.6)$ & & $35(0.4)$ & $242(2.4)$ & 0 & $51(0.5)$ & $130(1.3)$ & 0 & \\
\hline Possibly & $210(2.1)$ & $211(2.1)$ & & $35(0.4)$ & $101(1)$ & $105(1.1)$ & $145(1.5)$ & $30(0.3)$ & $7(0.1)$ & \\
\hline I have no idea & $30(0.3)$ & $20(0.2)$ & & $25(0.3)$ & $15(0.2)$ & 0 & $5(0.1)$ & $6(0.1)$ & 0 & \\
\hline & Using auxiliar & oth-cleaning toc & ther than & toothbrushes h & s the benefits 0 & f reducing too & th decay and & gingival dise & ease? & \\
\hline I agree & $1666(16.7)$ & $2078(20.8)$ & $P<0.001$ & $140(1.4)$ & $690(6.9)$ & $454(4.5)$ & $1360(13.6)$ & $984(9.8)$ & $113(1.2)$ & $P<0.001$ \\
\hline I do not agree & $751(7.5)$ & $1718(17.2)$ & & $115(1.2)$ & $399(4)$ & $460(4.6)$ & $775(7.8)$ & 691(6.9) & $31(0.3)$ & \\
\hline Undecided & $420(4.2)$ & $861(8.6)$ & & $49(0.5)$ & $276(2.8)$ & $152(1.5)$ & $408(4.1)$ & 395(4) & 0 & \\
\hline Possibly & 439(4.4) & $860(8.6)$ & & $66(0.7)$ & 185(1.9) & $55(0.6)$ & $358(3.6)$ & $535(5.4)$ & 101(1) & \\
\hline I have no idea & $296(3)$ & $911(9.1)$ & & $49(0.5)$ & $507(5.1)$ & $255(2.6)$ & $196(2)$ & $181(1.8)$ & $20(0.2)$ & \\
\hline & & It is & gh to use & nly antibiotics $\mathrm{w}$ & en toothache $c$ & or gingivitis occ & curs? & & & \\
\hline I agree & $705(7.1)$ & $1820(18.2)$ & $P<0.001$ & $100(1)$ & $735(7.4)$ & $630(6.3)$ & $650(6.6)$ & $394(3.9)$ & $15(0.2)$ & $P<0.001$ \\
\hline I do not agree & $1210(12.1)$ & $2173(21.7)$ & & $79(0.8)$ & $504(5)$ & $270(2.7)$ & $1279(12.8)$ & $1115(11.2)$ & $135(1.4)$ & \\
\hline Undecided & 392(3.9) & $466(4.7)$ & & $49(0.5)$ & $272(2.7)$ & $36(0.4)$ & $261(2.6)$ & $226(2.3)$ & $15(0.2)$ & \\
\hline Possibly & $906(9.1)$ & $1208(12.1)$ & & $111(1.1)$ & $385(3.9)$ & $276(2.8)$ & $542(5.4)$ & $730(7.3)$ & $69(0.7)$ & \\
\hline I have no idea & $359(3.6)$ & $761(7.6)$ & & $80(0.8)$ & $161(1.6)$ & $164(1.6)$ & $365(3.7)$ & $321(3.2)$ & $31(0.3)$ & \\
\hline & & When a & thache or $\mathrm{g}$ & ingivitis occurs, & ou should defin & hitely visit the & dentist? & & & \\
\hline I agree & $3324(33.2)$ & $5872(58.7)$ & 0.001 & $332(3.3)$ & $1773(17.7)$ & $1340(13.4)$ & $3006(30.1)$ & $2485(24.9)$ & $258(2.6)$ & $P<0.001$ \\
\hline I do not agree & $137(1.4)$ & $272(2.7)$ & & $51(0.5)$ & $197(2)$ & $15(0.2)$ & $71(0.7)$ & $69(0.7)$ & $5(0.1)$ & \\
\hline Undecided & $51(0.5)$ & $135(1.4)$ & & $10(0.1)$ & $26(0.3)$ & $15(0.2)$ & $15(0.2)$ & $120(1.2)$ & $1(0)$ & \\
\hline Possibly & $5(0.1)$ & $124(1.2)$ & & $6(0.1)$ & $16(0.2)$ & $1(0)$ & 0 & 105(1.1) & $1(0)$ & \\
\hline I have no idea & $55(0.6)$ & $25(0.3)$ & & $20(0.2)$ & $45(0.5)$ & $5(0.1)$ & $5(0.1)$ & $7(0.1)$ & 0 & \\
\hline & Studie & low that disease & he mout & (especially gins & ival disease) is & linked to other & problems in & the body? & & \\
\hline I agree & $1545(15.5)$ & $3162(31.6)$ & $P<0.001$ & $99(1)$ & $969(9.7)$ & $804(8)$ & $1169(11.7)$ & $1481(14.8)$ & $183(1.8)$ & $P<0.001$ \\
\hline I do not agree & $588(5.9)$ & $904(9)$ & & $110(1.1)$ & $265(2.7)$ & $175(1.8)$ & $472(4.7)$ & $414(4.1)$ & $55(0.6)$ & \\
\hline Undecided & $557(5.6)$ & $782(7.8)$ & & $71(0.7)$ & $171(1.7)$ & $216(2.2)$ & $676(6.8)$ & $185(1.9)$ & $21(0.29$ & \\
\hline Possibly & $566(5.7)$ & $440(4.4)$ & & $79(0.8)$ & $251(2.5)$ & $36(0.4)$ & $245(2.5)$ & $394(3.9)$ & $1(0)$ & \\
\hline I have no idea & $316(3.2)$ & $1140(11.4)$ & & $60(0.6)$ & $401(4)$ & $145(1.5)$ & $535(5.4)$ & $312(3.1)$ & $5(0.1)$ & \\
\hline & & The $c$ & sse of dent & caries and gin & ival diseases is & microorganisn & & & & \\
\hline I agree & 1885(18.9) & $3004(30)$ & $P<0.001$ & $184(18)$ & $827(8.3)$ & $499(5)$ & $1519(15.2)$ & $1664(16.6)$ & 193(1.9) & $P<0.001$ \\
\hline I do not agree & $375(3.8)$ & $741(7.4)$ & & $56(0.6)$ & $241(2.4)$ & $50(0.5)$ & $339(3.4)$ & $410(4.1)$ & $20(0.2)$ & \\
\hline Undecided & $425(4.3)$ & $692(6.9)$ & & $55(0.6)$ & $307(3.1)$ & $166(1.7)$ & $324(3.2)$ & $250(2.5)$ & $16(0.2)$ & \\
\hline Possibly & $\begin{array}{l}447(4.5) \\
\end{array}$ & $953(9.5)$ & & $11(0.1)$ & $156(1.6)$ & $406(4.1)$ & $561(5.6)$ & $261(2.6)$ & $6(0.1)$ & \\
\hline I have no idea & $440(4.4)$ & $1038(10.4)$ & & $113(1.1)$ & $526(5.3)$ & $255(2.6)$ & $354(3.6)$ & $201(2)$ & $30(0.3)$ & \\
\hline Smoking has ne & ative effects & ral and teeth he & & & & & & & & \\
\hline I agree & $3269(32.7)$ & $5632(56.3)$ & $P<0.001$ & $243(2.4)$ & $1713(17.1)$ & $1244(12.4)$ & $2825(28.3)$ & $2619(26.2)$ & $253(2.5)$ & $P<0.001$ \\
\hline I do not agree & $160(1.6)$ & $407(4.1)$ & & $81(0.8)$ & $156(1.6)$ & $115(1.2)$ & $70(0.7)$ & $140(1.4)$ & $5(0.1)$ & \\
\hline Undecided & $66(0.7)$ & $51(0.5)$ & & $25(0.3)$ & $25(0.3)$ & $11(0.1)$ & $46(0.5)$ & $10(0.1)$ & $1(0)$ & \\
\hline Possibly & $41(0.4)$ & $79(0.8)$ & & $36(0.4)$ & $42(0.4)$ & $1(0)$ & $26(0.3)$ & $11(0.1)$ & $6(0.1)$ & \\
\hline I have no idea & $36(0.4)$ & $259(2.6)$ & & $34(0.3)$ & $121(1.2)$ & $5(0.1)$ & $130(1.3)$ & $6(0.1)$ & 0 & \\
\hline Gingival bleedin & is the most & tant symptor & ngival dis & ase? & & & & & & \\
\hline I agree & $2265(22.7)$ & $5214(52.2)$ & $P<0.001$ & $183(1.8)$ & $1478(14.8)$ & $1165(11.7)$ & $2252(22.5)$ & $2175(21.8)$ & $223(2.2)$ & $P<0.001$ \\
\hline I do not agree & $481(4.8)$ & $595(6)$ & & $121(1.2)$ & $279(2.8)$ & $180(1.8)$ & $261(2.6)$ & $210(2.1)$ & $25(0.2)$ & \\
\hline Undecided & $364(3.6)$ & $116(1.2)$ & & $45(0.5)$ & $66(0.7)$ & $20(0.2)$ & $259(2.6)$ & $90(0.9)$ & $1(0)$ & \\
\hline Possibly & $215(2.2)$ & $268(2.7)$ & & $26(0.3)$ & $41(0.4)$ & $1(0)$ & $140(1.4)$ & $270(2.7)$ & $6(0.1)$ & \\
\hline I have no idea & $247(2.5)$ & $235(2.4)$ & & $44(0.4)$ & 193(1.9) & $10(0.1)$ & $185(1.9)$ & $41(0.4)$ & $10(0.1)$ & \\
\hline Diseases such a & hepatitis B an & IDS may give & oms in $\mathrm{tl}$ & mouth? & & & & & & \\
\hline I agree & $1070(10.7)$ & $1235(12.4)$ & 0.001 & $129(1.2)$ & $430(4.3)$ & $316(3.2)$ & $740(7.4)$ & $635(6.4)$ & $54(0.5)$ & $P<0.001$ \\
\hline I do not agree & $483(4.8)$ & 988(9.9) & & $45(0.5)$ & $389(3.9)$ & $280(2.8)$ & $317(3.2)$ & $424(4.2)$ & $16(0.2)$ & \\
\hline Undecided & $402(4)$ & $621(6.2)$ & & $91(0.9)$ & $196(2)$ & $70(0.7)$ & $390(3.9)$ & $241(2.4)$ & $34(0.3)$ & \\
\hline Possibly & $430(4.3)$ & $919(9.2)$ & & $39(0.4)$ & $285(2.9)$ & $115(1.2)$ & $360(3.6)$ & $550(5.5)$ & $1(0)$ & \\
\hline I have no idea & 1187(11.9) & $2665(26.7)$ & & $115(1.2)$ & $757(7.6)$ & $595(6)$ & $1290(12.9)$ & $936(9.4)$ & $160(1.6)$ & \\
\hline The mos & & & & se is to do & & & & & & \\
\hline I agree & $2910(29.1)$ & $5418(54.2)$ & 0.002 & $272(2.7)$ & $1728(17.3)$ & $1214(12.1)$ & $2507(25.1)$ & $2451(24.5)$ & $218(2.2)$ & $P<0.001$ \\
\hline I do not agree & $420(4.2)$ & $587(5.9)$ & & $116(1.2)$ & $286(2.9)$ & $40(0.4)$ & $265(2.7)$ & $263(2.6)$ & $36(0.4)$ & \\
\hline Undecided & $70(0.7)$ & $77(0.8)$ & & $10(0.1)$ & $32(0.3)$ & $15(0.2)$ & $69(0.7)$ & $21(0.2)$ & 0 & \\
\hline Possibly & $151(1.5)$ & $151(1.5)$ & & 0 & $10(0.1)$ & $101(1)$ & 141(1.4) & $40(0.4)$ & $11(0.1)$ & \\
\hline I have no idea & $21(0.2)$ & $132(1.3)$ & & $21(0.2)$ & $1(0)$ & $6(0.1)$ & $115(1.2)$ & $11(0.1)$ & 0 & \\
\hline Halitosis may b & symptom o & oblem in the & & & & & & & & \\
\hline I agree & $2550(2.6)$ & $4810(48.1)$ & 0.002 & $203(2)$ & $1419(14.2)$ & $944(9.4)$ & $2403(24)$ & $2174(21.7)$ & $213(2.1)$ & 0.001 \\
\hline I do not agree & $460(4.6)$ & $630(6.3)$ & & $115(1.2)$ & $189(1.9)$ & 191(1.9) & $255(2.6)$ & $305(3.1)$ & $35(0.4)$ & \\
\hline Undecided & $130(1.3)$ & $127(1.3)$ & & $50(0.5)$ & $72(0.7)$ & $15(0.2)$ & $115(1.2)$ & $5(0.1)$ & 0 & \\
\hline Possibly & $351(3.5)$ & $710(7.1)$ & & $20(0.2)$ & $291(2.9)$ & $210(2.1)$ & $250(2.5)$ & $291(2.9)$ & $1(0)$ & \\
\hline I have no idea & $81(0.8)$ & $151(1.5)$ & & $31(0.3)$ & $86(0.9)$ & $16(0.2)$ & $74(0.7)$ & $11(0.1)$ & $16(0.2)$ & \\
\hline
\end{tabular}




\begin{tabular}{|c|c|c|c|c|c|c|c|c|c|c|}
\hline \multicolumn{11}{|c|}{ Halitosis may be a symptom of a problem in the mouth? } \\
\hline agree & $2550(2.6)$ & $4810(48.1)$ & \multirow[t]{5}{*}{0.002} & $203(2)$ & $1419(14.2)$ & $944(9.4)$ & $2403(24)$ & $2174(21.7)$ & $213(2.1)$ & \multirow[t]{5}{*}{0.001} \\
\hline do not agree & $460(4.6)$ & $630(6.3)$ & & $115(1.2)$ & $189(1.9)$ & 191(1.9) & $255(2.6)$ & $305(3.1)$ & $35(0.4)$ & \\
\hline Indecided & $130(1.3)$ & $127(1.3)$ & & $50(0.5)$ & $72(0.7)$ & $15(0.2)$ & $115(1.2)$ & $5(0.1)$ & 0 & \\
\hline Possibly & $351(3.5)$ & $710(7.1)$ & & $20(0.2)$ & $291(2.9)$ & $210(2.1)$ & $250(2.5)$ & $291(2.9)$ & $1(0)$ & \\
\hline have no idea & $81(0.8)$ & $151(1.5)$ & & $31(0.3)$ & $86(0.9)$ & $16(0.2)$ & $74(0.7)$ & $11(0.1)$ & $16(0.2)$ & \\
\hline \multicolumn{11}{|c|}{ Halitosis may be caused by another disease? } \\
\hline agree & $2123(21.2)$ & $3121(31.2)$ & \multirow[t]{5}{*}{$\mathrm{P}<0.001$} & $154(1.5)$ & $900(9)$ & $1005(10.1)$ & $1425(14.3)$ & $1584(15.8)$ & $173(1.7)$ & \multirow[t]{5}{*}{0.002} \\
\hline do not agree & $710(7.1)$ & $685(6.9)$ & & $116(1.2)$ & $289(2.9)$ & $75(0.8)$ & $450(4.6)$ & $420(4.2)$ & $45(0.5)$ & \\
\hline Indecided & $337(3.4)$ & $766(7.7)$ & & $69(0.7)$ & $528(5.3)$ & $126(1.3)$ & $300(3)$ & $74(0.7)$ & $6(0.1)$ & \\
\hline Possibly & 101(1) & $1306(13.1)$ & & $24(0.2)$ & $170(1.7)$ & $120(1.2)$ & $582(5.8)$ & $511(5.1)$ & $1(0)$ & \\
\hline have no idea & $301(3)$ & $550(5.6)$ & & $56(0.6)$ & $170(1.7)$ & $50(0.5)$ & $340(3.4)$ & $197(2)$ & $40(0.4)$ & \\
\hline \multicolumn{11}{|c|}{ Nutrition has an effect on oral and dental health? } \\
\hline agree & $2859(28.6)$ & $4130(41.3)$ & \multirow[t]{5}{*}{$\mathrm{P}<0.001$} & 193(1.9) & $1259(12.6)$ & $864(8.6)$ & $2157(21.6)$ & $2284(22.8)$ & $228(2.3)$ & \multirow[t]{5}{*}{$\mathrm{P}<0.001$} \\
\hline do not agree & $376(3.8)$ & $609(6.1)$ & & $91(0.9)$ & $304(3)$ & $155(1.6)$ & $230(2.3)$ & $170(1.7)$ & $35(0.4)$ & \\
\hline Indecided & $146(1.5)$ & $210(2.1)$ & & $59(0.6)$ & $107(1.1)$ & $25(0.3)$ & $95(1)$ & $71(0.8)$ & 0 & \\
\hline Possibly & $50(0.5)$ & $1223(12.2)$ & & $45(0.5)$ & $276(2.8)$ & $227(2.3)$ & $510(5.1)$ & $215(2.2)$ & $2(0)$ & \\
\hline have no idea & $141(1.4)$ & $256(2.6)$ & & $31(0.3)$ & $111(1.1)$ & $105(1.1)$ & $105(1.1)$ & $46(0.5)$ & 0 & \\
\hline \multicolumn{11}{|c|}{ Regular oral and dental examinations should be done at least six-monthly intervals? } \\
\hline agree & $2495(25)$ & $4900(49)$ & \multirow[t]{5}{*}{$\mathrm{P}<0.001$} & $209(2.1)$ & $1446(14.5)$ & $1054(10.5)$ & 2091(20.9) & $2355(23.6)$ & $238(2.4)$ & \multirow[t]{5}{*}{$\mathrm{P}<0.001$} \\
\hline do not agree & $425(4.3)$ & $515(5.2)$ & & $51(0.5)$ & 184(1.8) & $160(1.6)$ & $345(3.5)$ & 184(1.8) & $16(0.2)$ & \\
\hline Indecided & $245(2.5)$ & $335(3.4)$ & & $50(0.5)$ & $270(2.7)$ & $30(0.3)$ & $230(2.3)$ & 0 & 0 & \\
\hline Possibly & $231(2.3)$ & $422(4.2)$ & & $50(0.5)$ & $66(0.7)$ & 121(1.2) & $235(2.4)$ & 171(1.7) & $11(0.1)$ & \\
\hline have no idea & $176(1.8)$ & $256(2.6)$ & & $59(0.6)$ & $91(0.9)$ & $11(0.1)$ & $196(2)$ & $76(0.8)$ & 0 & \\
\hline
\end{tabular}

The data were analysed using $X^{2}$ tests, $P<0.05$.

Tablo 5. Association of periodontal status with demographic characteristics of patients

\begin{tabular}{|c|c|c|}
\hline \multirow[t]{2}{*}{ Variable } & \multicolumn{2}{|c|}{ Periodontal Status $\mathrm{OR}^{\mathrm{a}}(95 \% \mathrm{CI})$} \\
\hline & Gingivitis & Periodontitis \\
\hline \multicolumn{3}{|l|}{ Gender } \\
\hline $\begin{array}{l}\text { Female(ref) } \\
\text { Male }\end{array}$ & $0.75(0.69-0.82)^{* * *}$ & $1.33(1.23-1.45)^{* * *}$ \\
\hline \multicolumn{3}{|l|}{ Age (years) } \\
\hline \multicolumn{3}{|l|}{$18-30$ (ref) } \\
\hline $31-43$ & $0.47(0.35-0.69)^{* * *}$ & 1.64(1.13-2.01) \\
\hline $44-56$ & $0.22(0.19-0.31)^{* * *}$ & $1.73(1.41-2.53)^{* *}$ \\
\hline $57-69$ & $0.24(0.16-0.37)^{* * *}$ & $1.71(1.33-2.35)^{* *}$ \\
\hline $70-82$ & $2.01(0.78-5.20)$ & $0.64(0.45-0.83)^{* *}$ \\
\hline \multicolumn{3}{|l|}{ Level of Education } \\
\hline \multicolumn{3}{|c|}{ None (pre-school) (ref) } \\
\hline Elementary school & $0.37(0.30-0.46)^{* * *}$ & $1.36(1.07-1.78)$ \\
\hline Middle school & $0.25(0.20-0.32)^{* * *}$ & $1.44(1.71-2.94)^{* * *}$ \\
\hline High school & $1.32(1.07-1.62)^{*}$ & $0.90(0.72-1.01)^{* * *}$ \\
\hline University & $1.15(0.93-1.42)$ & $0.95(0.79-1.27)^{* *}$ \\
\hline Phd or Above & $0.83(0.61-1.14)$ & $1.08(0.81-1.14)^{*}$ \\
\hline
\end{tabular}

\footnotetext{
CI, confidence interval; OR, crude odds ratio
}

a Model included gender, age and education level

The data were analysed using logistic regression models for complex survey samples.

The $P$ values are marked as statistically significant. ${ }^{*} p<0.05, * * p<0.01, * * * p<0.001$

Those who were graduated from high school and university were found to be at more risk of gingivitis (OR:1.32, 95\% CI:1.07-1.62, OR:1.15, 95\% CI:0.931.42 , respectively) compared to those who were only literate. However, those graduated from primary and secondary school were found to be at more risk of gingivitis (OR:1.36, \%95 CI:1.07-1.78, OR: 1.44,\%95 $\mathrm{CI}: 1.71-2.94$, respectively) compared to those who were only literate. The estimated validity of the questions in the model was tested by calculating sensitivity and specificity values. The sensitivity value was $58.5 \%$, and the specificity value was $78.2 \%$ (Table 5).

\section{DISCUSSION}

The criteria that show the level of $\mathrm{ODH}$ in societies are also information that is comparable to groups and geographical regions. Basic ODH research 
is needed to reach country-level or regional criteria. Oral and dental diseases can be prevented with good oral hygiene. Treatment of oral and dental diseases is expensive for both developed and developing countries. Oral and dental health expenses for individuals and governments can be reduced by the application of protective programs ${ }^{12}$. After the emergence of possible relations between various diseases and $\mathrm{ODH}$ through the conducted studies, researchers aimed to investigate the status of $\mathrm{ODH}$ in large populations. In addition to clinical studies for this purpose, survey studies are also available ${ }^{13,14}$.

The use of surveys in obtaining data for research is an easier and lower-cost method and has a supportive effect on the arrangement of oral health programs ${ }^{15}$. In our study, a face-to-face interview method was chosen to assess the ODH awareness of patients admitted to our clinic, and the questions were explained to the participants to be better understood, and answers were filled by the researchers. This study may not completely disclose the level of knowledge about the ODH of the patients in the entire southeast region of Turkey due to the limited population size, but it can be a guide for information about the $\mathrm{ODH}$ of adult patients in this region. In previous studies, Eren et al. ${ }^{16}$ studied on 187 patients and reported the proportion of periodontitis and gingivitis in our society as $30.5 \%$ and $69.5 \%$, respectively; Gökalp et al. ${ }^{6}$ reported as $34.1 \%$ and $35.9 \%$, respectively, in 3176 patients aged 35-74; and Ertümer et al. ${ }^{17}$ reported as $28.3 \%$ and $57.7 \%$, respectively, in 350 patients, while we found these numbers as $64.3 \%$ and $35.7 \%$, respectively. The higher rate of periodontitis found in the present study may be due to the fact that the total number of individuals is more, or due to the extended age range, or due to the limited region.

To prevent tooth decay and gingivitis, it was stated that brushing teeth at least twice a day and using an auxiliary tooth-cleaning tool at least once a day were important ${ }^{18,19}$. In a study, brushing teeth at least twice a day in Japanese society was reported as $70 \%{ }^{20}$. Saito et al. ${ }^{11}$ reported that the rate of those brushing their teeth at least twice a day and using auxiliary tooth-cleaning tools was $60 \%$, while, similar to our study, Gökalp et al. ${ }^{6}$ reported this rate as $34.7 \%$ in adult patients. Also, Behram et al. ${ }^{21}$ reported the proportion of users that use the auxiliary cleaning tools as $48 \%$. In the present study, $80.4 \%$ and $37.4 \%$ of the patients agreed on these two statements; "Teeth should be brushed for 2 minutes at least 2 times a day?" and "Using auxiliary cleaning tools other than a toothbrush has benefits in reducing tooth decay and gingival disease?". The fact that most of the patients in the present study agreed on the benefits of brushing teeth at least two times one day and the fact that our patient's mean age is much are related to the increase of awareness of teeth health, as said by Gökalp et al ${ }^{6}$. Also, $21.2 \%$ of women, $16.2 \%$ of patients in $18-30$ age group, $20.8 \%$ of patients with periodontitis and $13.6 \%$ of high school graduated patients acknowledged the statement about the auxiliary cleaning tools other than a toothbrush. Gökalp et al. ${ }^{6}$ stated that the proportion of those using auxiliary cleaning tools in the 35-44 age group was $9.2 \%$, and, in our study, the proportion in the 31 43 age group was $12.2 \%$.

It was reported that women care more about dental cleaning practices than men ${ }^{22}$. In our study, women are more concerned with dental care, similar to the mentioned study. Most of the patients who agreed on the statement "I brush my teeth at least twice a day" were mostly women (45.1\%) and young people in the 18-30 age group (35.5\%). Gökalp et al. 6 reported the proportion of those brushing teeth at least twice a day as $22.2 \%$ in the $35-44$ age group. In a study in Chinese society, this rate is $32 \%$ in the $35-$ 44 age group ${ }^{23}$. In the present study, it was found to be $27.6 \%$ in $31-43$ age group, which is close to the mentioned ratio. The participants who brushed their teeth at least twice a day had periodontitis in the rate of $56.4 \%$ and $27.2 \%$ of whom were graduated from high school. Teker et al. ${ }^{24}$ reported the highest proportion of those brushing teeth at least once a day as $82.1 \%$ in people graduated from high school, which is compatible with the findings of our study. Ertümer et al. ${ }^{17}$ reported the rate of those who brushed their teeth at least twice a day as $10 \%$, and Eren et al. 16 reported the rate of those with periodontitis as $40.4 \%$, which are different from the present study. This can be explained by the fact that our work has a large population volume and wider field work.

In the findings of a research conducted by Güngör et al ${ }^{25}$, which overlapped with the findings of our study, the level of education and giving importance by individuals to oral health were determined to show a parallel increase. As the level of education increased, oral and dental health care was also improved, and this finding was interpreted by the increase in the level of education in patients and the increase in the tendency to go to a physician. 
In a study conducted in China, the rate of those who visited the dentists only if they had a toothache was determined as $74 \%$, while this rate was reported as $\% 88$ by Özbek et al ${ }^{27}$ and Gökalp et al ${ }^{6}$. In the present study, this number is $91.9 \%$, which is consistent with these two studies conducted in our country. The majority of patients do not go to dentist control unless they have complaints. This situation leads to failure of preventive dentistry practices and causes late diagnosis and treatment. We think it would be a great advantage to apply preventive dentistry practices on the $\mathrm{ODH}$ by dentists.

Antibiotics, which are among the most consumed drugs in our country, have a very wide usage area in dentistry applications ${ }^{28}$. The percentage of antibiotic use in Nigeria was $21.2 \%{ }^{29}$, while a study conducted in different provinces of Turkey indicated that there was a significant decrease in the percentage of antibiotic use without examination by the dentist. Also, $51.9 \%$ of the individuals in Adana province ${ }^{30}$ and $75.5 \%$ in Ankara province ${ }^{31}$ were using self-medication in Ankara. In our study, the distribution of the ratio of those who use only antibiotics for toothache and gingivitis was $12.7 \%$ male and $12.6 \%$ female, $10.5 \%$ in the age group of $31-43$ years, $18.2 \%$ those with periodontitis and $7.4 \%$ those graduated from primary schools. In a study performed by Koyuncuoglu et al. ${ }^{32}$, the rate of men who used antibiotics without examination by the dentist was $3.24 \%$ and the rate of women was $5.12 \%$ and for those graduated from primary school, it was $6.86 \%$. Similarly, it was reported that self-medication is higher in women than in men ${ }^{33}$. It can be said that women are generally more prone to self-medication than men for dental reasons; however, in our study, the rates of men and women were found to be similar in contradistinction to those in the literature. These results may be due to the extended and different working area of our study. When the literature is examined, it is determined that the rate of selfmedication is high in the groups with high educational level ${ }^{34,35}$. This coincides with our study results.

In our research, $38.5 \%$ of patients stated to have no knowledge about the statement "Diseases such as hepatitis B and AIDS can give symptoms in the mouth", this rate is quite high. The incidence of hepatitis B from these two systemic disorders is observed at a high rate of $2 \%-7 \%$ in our country ${ }^{36}$, and the prevalence of HIV/AIDS is $0-0.13 \%{ }^{37}$. It can be aimed to increase the level of consciousness of society by organizing various informational programs to inform the community about these systemic diseases whose mode of transmission are similar.

Smoking has been reported to cause tooth discoloration, gingival diseases, inflammation of the oral mucosa, alveolar bone loss and oral hygiene disorder in the mouth ${ }^{38}$. In our study, those who acknowledge the statement "Smoking negatively affects oral and tooth health?" is $89 \%$. These results are higher than the results in the study of Stepteo et al. ${ }^{39}$. The high rate of this ratio may be due to that the government bans smoking in confined spaces in our country and due to the spot announcements on this issue.

The main clinical symptom of periodontal diseases is bleeding in the gingiva. In developing and underdeveloped societies, it was observed that people were not very aware of these symptoms ${ }^{40}$. Therefore, $74.8 \%$ of the participants agreed on the statement "Gingival bleeding is the most important symptom of the gingival disease?". The highest rates of this statement are $40.9 \%$ in women, $29.1 \%$ in those in the 18-30 age group, $52.2 \%$ in those with periodontitis and $22.5 \%$ in those graduated from high schools. Taani et al. ${ }^{41}$ reported that $25.6 \%$ of individuals had the bleeding in their gingiva and $58.9 \%$ of them were women. In the present study, women have a high awareness of gingival bleeding, oral hygiene, and care. Al-Qaderi and Ta'ani (42) stated that the individuals in the young age group (age 20-29 and 30-39) complained more about the bleeding of gingiva compared to the elderly (40-90 and 50-60 age), which overlaps with our study findings, but not consistent with the study of Pekiner et al. ${ }^{43}$. Yilmaz et al. ${ }^{44}$ reported the proportion of those who were aware of gingival bleeding as 51.82 $\%$, and $79 \%$ of them were graduated from primary school. The findings of our study are consistent with the study of Yilmaz et al. ${ }^{44}$. In our study, the awareness that gingival bleeding was a symptom of disease changed according to the education status of the people, which suggests that adequate health information exists throughout society.

Halitosis is a very common problem affecting people of all ages. When it is severe or prolonged, it can cause a decrease in self-confidence and social interactions. Halitosis is sourced from the inside of the mouth at the rate of $87-90 \%$. Therefore, oral halitosis is a very common condition in patients ${ }^{45,46}$. A study in Jordan reported that $20.5 \%$ of the patients are aware 
that halitosis is a problem in the mouth ${ }^{47}$. In our study, $73.6 \%$ of the participants expressed their knowledge about there is a problem in the mouth if halitosis occurs. This suggests that our patient groups have a high level of consciousness about the halitosis.

It is very important for individuals to have regular dentist check-ups for protection from $\mathrm{ODH}$. In addition, it varies depending on the regular controls by dentists, each individual's oral health status and the risk factors of diseases. Vano et al. ${ }^{48}$ reported that in the Italian adult society, $23 \%$ of patients in total (29 $\%$ of women and $18 \%$ of men) visited a dentist every 6 months. Gökalp et al. ${ }^{6}$ reported that the rate of individuals visiting a dentist at least once a year was $46.2 \%$ in the $35-44$ age group, while $20.2 \%$ in the 65-74 age group. In our study, women and individuals in the age group of $13-30$ agreed on that the statement 'regular oral and dental examination should be at least six-monthly intervals' in the highest rate of $40.6 \%$ and $31.8 \%$, respectively. In another study, individuals of 45 years old and older stated to go to a dentist examination at a lesser rate than 25-34 and 35-44 age groups ${ }^{49}$. Those with periodontitis and graduate-level education stated also to agree on that, they showed the highest rate (49\% and $23.9 \%$, respectively). As a result, regular dental examinations in young and highly educated individuals demonstrate that their $\mathrm{ODH}$ care is better in accordance with the literature findings.

In most of the research that examines the relationship between periodontal disease and gender, it has been shown that male individuals have a higher severity of periodontal disease ${ }^{50,51}$. Because of this, gender differences in the prevalence and severity of periodontal disease are considered to be associated with the care and habits of oral health rather than $a$ genetic factor. In our study, there was a difference between the periodontal states of male and female individuals, and men were found to be at risk of 1.33 times more (95\% CI: 1.23-1.45) than women in terms of having periodontitis. In the study of Ertümer et al. ${ }^{17}$, men were found to be at risk of 4.34 times more (95\% CI: 1.04-10.90) than women in terms of having periodontitis. According to the study conducted in Korean society, males were found to be at risk of 1.78 times more (95\% CI: 1.56-2.03) than women in terms of having periodontitis ${ }^{52}$. This similarity may be due to the fact that the individuals included in our study were selected among patients who applied to the faculty of dentistry.
In our study, the prevalence of gingivitis and periodontitis was determined as $35.7 \%$ and $64.3 \%$, respectively. Studies have shown that the severity and prevalence of periodontal disease increase with age ${ }^{53}$. This condition can be attributed to the increased severity of periodontal disease with age due to the chronic character of periodontal tissues, duration of exposure to bacterial plaque, and cumulative oral history ${ }^{17,53}$. Genco et al. ${ }^{54}$ stated that self-notification measurements of the disease were predictable because of age that is an important risk factor for periodontitis. Ertümer et al. ${ }^{17}$ reported that the percentage of gingivitis in those under 30 years was more than other age groups, whereas the percentage of having periodontitis was lower, also, 31-45 age group were at a risk of 7.5 times more (95\% CI: 3.44-22.20) than those under 30 years old in terms of having periodontitis, and those over the age of 45 were found at a risk of 9.7 times (95 \% CI: 3.8528.63) more than those under the age of 30 . In the study of Lee et al. ${ }^{52}, 45-54$ and 55-64 age groups were determined to be at risk of 2.91 times (95\% CI: 2.33-3.63) and 3.09 times more (95 \% CI: 2.36-4.04), respectively, in terms of periodontitis compared to the 19-24 age group. Similarly, in our study, 57-69 age groups were determined to be at risk of 0.24 times (95 \% CI: 0.16-0.37) and 1.71 times more (95 \% CI: 1.33-2.35) compared to the 18-30 age group in terms of gingivitis and periodontitis, respectively.

According to NHANES 1999-2004, there is a significant relationship between the education level and periodontal disease of adult individuals older than 18 years of age ${ }^{55}$. Similarly, Haas et al. ${ }^{56}$ and Richard et al. ${ }^{57}$ reported that the level of education was associated with the development of the periodontal disease. In the study of Lee et al. ${ }^{52}$, those with a high level of education were found to be at risk of 2.06 times more (95\% CI: 1.47-2.89) compared to those with low levels of education in terms of periodontal disease. Ertümer et al. ${ }^{17}$ stated that the variable of education level was found to be significant in univariate analysis, but was not significant in multivariate analysis. In our study, the risk of periodontal disease increases as the level of education increases, which is in accordance with the study of Lee et al ${ }^{52}$. These findings suggest that patients with high education status are aware of oral hygiene and oral care related tasks, but do not have oral hygiene behaviors. 


\section{CONCLUSION}

The use of $\mathrm{ODH}$ self-notification surveys can be considered an effective and economical tool for major epidemiological studies. According to the survey results, the majority of respondents are aware that oral and dental health is important, but we think that individuals do not give importance on oral care to protect oral and dental health because of the high prevalence of the periodontal disease. It was determined that the patients were going to the dentist only when they had a toothache and that they did not adopt regular dentist control. As a result, patients should be informed by the dentist and be warned about not waiting for a toothache complaint to go to the dentist. Age, gender and education level affect $\mathrm{ODH}$ awareness and factors such as periodontal disease. These factors should be considered when dentists are taking preventive measures and planning treatment.

In our society, to inform about the importance of oral health in the education system, and to raise awareness of individuals through the media are very important issues. At the same time, society should be informed about a regular oral and dental examination, and urgent development of programs that will make the awareness of individuals in the community about oral and dental health to be transformed into behaviors should be provided.

\section{Acknowledgements}

The authors declare that there were no other contributors involved in this work.

Conflicts of interest statement

The authors declare no conflict of interest.

\section{REFERENCES}

1. Ramirez $\mathrm{JH}$, Arce $\mathrm{R}$, Contreras A. Why must physicians know about oral diseases? Teaching and Learning in Medicine 2010; 22:148-55.

2. Peterson EP. The World Oral Health Report 2003: Continuous İmprovement of Oral Health in The 21st Century-The Approach of the WHO Global Oral Health Programme. Community Dent Oral Epidemiol 2003; 31: 3-24.

3. Cortes FJ, Nevot C, Ramon JM, et.al. The Evolution of Dental Health in Dental Students at University of Barcelona. J Dent Educ 2002; 66: 1203-8.
4. Kulak-Özkan Y, Özkan Y, Kazazoğlu E, Arıkan A. Dental caries prevalence, tooth brushing and periodontal status in 150 young people in Istanbul: A pilot study. Int Dent J 2001; 51: 4516.

5. Sogaard AJ, Tuominen R, Holst D, Gjermo P. The effect of 2 teaching programmes on the gingival health of 15 - year old school children. J Clin Periodontol 1987;14: 165-70,

6. Gokalp $S$, et al. The oral health profile of adults and elderly, Turkey-2004. J Hacettepe Faculty Dent 2007; 31: 11-8.

7. Villalobos-Rodelo JJ, Medina-Solis CE, Maupome G, Vallejos- Sanchez AA, Lau-Rojo L, Leon-Viedas PL. Socioeconomic and sociodemographic variables associated with oral hygiene status in Mexican schoolchildren aged 6 to 12 years. J Periodontol 2007;78: 816- 22.

8. Mengel $\mathrm{R}$, Koch $\mathrm{H}$, Pfeifer $\mathrm{C}$, Flores de Jacoby $\mathrm{L}$. Periodontal health of the population in eastern Germany. J Clin Periodontol 1993;20: 752-5.

9. Löe $\mathrm{H}$ : Oral hygiene in the prevention of caries and periodontal disease. Int Dent J 2000; 50:129-39.

10. Renz A, Ide M, Newton T, Robinson PG, Smith D: Psychological interventions to improve adherence to oral hygiene instructions in adults with periodontal diseases. Cochrane Database Syst Rev 2007; 18:CD005097.

11. Saito, A., Kikuchi, M., Ueshima, F., Matsumoto, S., Hayakawa, H., Masuda, H., \& Makiishi, T. Assessment of oral self-care in patients with periodontitis: a pilot study in a dental school clinic in Japan. BMC Oral Health, 2009:9: 27.

12. Petersen PE, Bourgeois D, Bratthall D, Ogawa $H$. Oral health information systems-towards measuring progress in oral health promotion and disease prevention. Bulletin of the World Health Organization 2005; 83: 686-93.

13. Palmqvist $S$, Soderfeldt B, Arnbjerg D. Dental conditions in a Swedish population aged 45-69 years. A questionnaire study. Acta Odontol Scand 1991; 49: 377-84.

14. Bormann C. Are self-reported diseases reliable and plausible? Problems in the estimation of the prevalence of heart infarct using the questionnaires data from the National Health Survey. Soz Praventivmed 1994; 39: 67-74. 
15. Kallio P. Self-assessed bleeding in monitoring gingival health among adolescents. Community Dent Oral Epidemiol 1996; 24: 128-32.

16. Eren, Gülnihal, and Sema Becerik. "Evaluating the Periodontal Status and Oral Hygiene Habits Among Dental Patients." Istanbul Medical Journal 2016;17:93-8

17. Ertümer, Ezgi, Fatma Karacaoğlu, and Murat Akkaya. Evaluation of periodontal disease selfreport validty of patients attended to Ankara University Dentistry Faculty. Selcuk Dental Journal 2018;5: 39-49.

18. Ramsay DS. Patient compliance with oral hygiene regimens: a behavioral self-regulation analysis with implications for technology. Int Dent J 2000;50:304-11.

19. Kressin NR, Boehmer $U$, Nunn ME, Spiro A. Increased preventive practices lead to greater tooth retention. J Dent Res 2003;82:223-7.

20. Dental Health Division of Health Policy Bureau, Ministry of Health and Welfare, Japan: Report on the survey of dental diseases Dental Health Division of Health Policy Bureau, Ministry of Health and Welfare, Japan; 2005.

21. Behram Ö, Lofça G, Güray Efes B. Correlation Between DMFT- DMFS Indices, Salivary and Microbiological Tests of The Patients Who Applied Department of Operative Dentistry. Journal of Istanbul University Faculty of Dentistry 2011; 45: 29-36.

22. Ashley P. Toothbrushing: Why, when and how? Dental Update 2001; 28: 36-40.

23. Zhu L, Petersen PE, Wang HY, Bian JY, Zhang BX. Oral health knowledge, attitudes and behaviour of adults in China. International dental journal 2005;55: 231-41.

24. Teker B, Ağadayı E, Küçükceran H, Karaca SN, Aydın S. Knowledge and behaviors about oral health of adults admitted to family medicine clinic in a rural area. Turkish Journal of Family Practice 2018; 22: 193-201.

25. Güngör K, Tüter G, Bal B: The evalucation of the relationship between educational status/ Eğitim düzeyi ile ağız sağığı arasındaki ilişkinin değerlendirilmesi. GÜ Diş Hek Fak Derg 1999; 16: 21- 5.

26. Rong WS, Wang WJ, Yip HK. Attitudes of dental and medical students in their first and final years of undergraduate study to oral health behaviour. Eur J Dent Educ 2006;10:178-84.
27. Özbek $C D$, Eser D, Bektaş Kayhan K, Ünür $M$. Comparison of the tooth brushing habits of primary school age children and their parents. Journal of Istanbul University Faculty of Dentistry 2015; 49: 33-40.

28. Baig QA, Muzaffar D, Afaq A, Bilal S, Iqbal N. Prevalence of self medication among dental patients. PODJ 2012;32: 292-5.

29. Agbor MA, Azodo CC. Self medication for oral health problems in Cameroon. Int Dent J 2011;61: 204-9.

30. Pınar N, Karataş Y, Bozdemir N, Ünal İ. Medicine Use Behaviors of People in the City of Adana. TAF Prev Med Bull 2013; 12:639-50.

31. Özçelikay G. A Pilot Study On Rational Drug Use. J Fac Pharm, Ankara, 2001;30: 9-18.

32. Koyuncuoğlu CZ, Kırmızı Nİ, Ceylan İ, AkıCı A. Investigation of Patients' Attitudes Regarding Drug Use Before Application to Dental Clinics. Marmara Pharml J 2017; 21: 165-76.

33. Karabay $\mathrm{O}$, Hosoglu S. Increased antimicrobial consumption following reimbursement reform in Turkey. J Antimicrob Chemother 2008;61:116971.

34. Nunes de Melo $M$, Madureira $B$, Nunes Ferreira AP, Mendes Z, Miranda Ada C, Martins AP. Prevalence of selfmedication in rural areas of Portugal. Pharm World Sci 2006;28: 19-25

35. Shankar PR, Partha P, Shenoy N. Self-medication and nondoctor prescription practices in Pokhara valley, Western Nepal: a questionnaire-based study. BMC FamPract 2002;3: 17.

36. Ozaslan M, Ozaslan E, Barsgan A, Koruk M. Mutations in the $S$ gene region of hepatitis $B$ virus genotype $D$ in Turkish patients. J Genet 2007; 86: 195-201.

37. Bulut $N$, Yenisehirli G, Bulut Y. Seroprevalence of hepatitis B, hepatitis C, HIV and syphilis in blood donors in Tokat. Viral Hepatit Dergisis 2012; 18:11-4

38. Steptoe A, Wardle J, Cui W, Baban A, Glass K, Karl $P$, et al. An international comparison of tobacco smoking, beliefs and risk awareness in university students from 23 countries. Addiction 2002;97:1561-71.

39. Bergström J, Eliasson S, Dock J. A 10-Year prospective study of tobacco smoking and periodontal health. J Periodontol 2000; 71:133847. 
40. Lin HC, Wong MC, Wang ZJ, Lo EC. Oral health knowledge, attitudes, and practices of Chinese adults. J Dent Res 2001; 80: 1466-70.

41. Taani DQ. Periodontal awareness and knowledge, and pattern of dental attendance among adults in Jordan. Int Dent J 2002; 52: 9498.

42. El-Qaderi SS, Ta'ani DQ. Assessment of periodontal knowledge and periodontal status of an adult population in Jordan. Int J Dent Hygiene 2004; 2: 132-6.

43. Pekiner F, Gumru B, Borahan MO, Aytugar E. Evaluation of demands and needs for dental care in a sample of the Turkish population. Eur J Dent 2010; 4: 143.

44. Yılmaz DB, Aykol-Şahin G, Başer Ü, Yalçın F, Onan U. Awareness of periodontal disease among the patients applied to Istanbul University Faculty of Dentistry DOI: 10.5505/yeditepe. 2016.20591.

45. Seemann $R$, Conceicao MD, Filippi $A$, Greenman J, Lenton $P$, Nachnani S, Quirynen M, Roldan S, Schulze $H$, Sterer N, Tangerman A, Winkel EG, Yaegaki K, Rosenberg M. Halitosis management by the general dental practitioner-results of an international consensus workshop. J Breath Res 2014;8: 017101.

46. McNab R. Oral malodour-a review. Arch Oral Biol 2008; 53: 1-7.

47. Hammad MM, Darwazeh AM, Al-Waeli H, Tarakji $B$, Alhadithy $\Pi$. Prevalence and awareness of halitosis in a sample of Jordanian population. J Int Soc Preventive \& Commun Dent 2014; 4: S178.

48. Vano M, Gennai S, Karapetsa D, Miceli M, Giuca $M R$, Gabriele M, Graziani F. The influence of educational level and oral hygiene behaviours on DMFT index and CPITN index in an adult Italian population: an epidemiological study. Int J Dent Hygiene 2015; 13: 151-7.

49. Lee MY, Chung WG, Kim CB, Kim NH. Analysis on the difference between needs and recipients for scaling and its trend in Korea (2000-2009). J Korean Acad Oral Health 2012; 36: 137-43.

50. Sezgin Y, Yılmaz Nişancl, MN. Evaluation of The Periodontal Status and Systemic Diseases of the Patients Referring to The Department of Periodontology. J Dent Fac Atatürk Uni 2019;29: 432-9.
51. Tanık, A. Evaluation of the relationship of CPITN and DMFT index of adult patients in Turkey with their demographic characteristics: an epidemiological study. Biotechnology \& Biotechnological Equipment 2019; 33:1626-34.

52. Lee MY, Chang SJ, Kim CB, Chung WG, Choi EM, Kim NH. Community periodontal treatment needs in South Korea. Int J Dent Hygiene 2015; 13: 254-60.

53. Genco RJ. Current view of risk factors for periodontal diseases. J Periodontol 1996; 67: 1041-9.

54. Genco RJ, Falkner KL, Grossi S, Dunford R, Trevisan M. Validity of self-reported measures for surveillance of periodontal disease in two western New York population-based studies. J Periodontol 2007; 78: 1439-54.

55. Borrell LN, Crawford ND. Social disparities in periodontitis among United States adults 19992004. Community Dent Oral Epidemiol 2008; 36: 383-91.

56. Haas AN, Gaio EJ, Oppermann RV, Rosing CK, Albandar JM, Susin C. Pattern and rate of progression of periodontal attachment loss in an urban population of South Brazil: a 5-years population-based prospective study. J Clin Periodontol 2012; 39: 1-9

57. Richard P GT, Chava V. Influence of lifestyle, gender and socioecomonic status determinants of dental health behaviour, periodontal status awareness. JPFA 2000; 14: 21-5.
Yazışma Adresi
Abdulsamet TANIK
Department of Periodontology
Faculty of Dentistry, Adıyaman University, Adıyaman, Turkey
Mobile phone:+905442611212
E-mail: samet.120a@gmail.com 